\title{
Sources and measurement of ultraviolet radiation
}

\author{
Brian L. Diffey* \\ Regional Medical Physics Department, Newcastle General Hospital, Newcastle upon Tyne NE4 6BE, UK
}

Accepted 19 June 2002

\begin{abstract}
Ultraviolet (UV) radiation is part of the electromagnetic spectrum. The biological effects of UV radiation vary enormously with wavelength and for this reason the UV spectrum is further subdivided into three regions: UVA, UVB, and UVC. Quantities of UV radiation are expressed using radiometric terminology. A particularly important term in clinical photobiology is the standard erythema dose $(S E D)$, which is a measure of the erythemal effectiveness of a UV exposure. UV radiation is produced either by heating a body to an incandescent temperature, as is the case with solar UV, or by passing an electric current through a gas, usually vaporized mercury. The latter process is the mechanism whereby UV radiation is produced artificially. Both the quality (spectrum) and quantity (intensity) of terrestrial UV radiation vary with factors including the elevation of the sun above the horizon and absorption and scattering by molecules in the atmosphere, notably ozone, and by clouds. For many experimental studies in photobiology it is simply not practicable to use natural sunlight and so artificial sources of UV radiation designed to simulate the UV component of sunlight are employed; these are based on either optically filtered xenon arc lamps or fluorescent lamps. The complete way to characterize an UV source is by spectroradiometry, although for most practical purposes a detector optically filtered to respond to a limited portion of the UV spectrum normally suffices. (C) 2002 Elsevier Science (USA). All rights reserved.
\end{abstract}

Keywords: Ultraviolet radiation; Spectrum; Radiometry; Standard erythema dose; Sunlight; Ultraviolet lamps; Solar simulator; Spectroradiometry; Dosimetry

\section{Introduction}

In 1666 Isaac Newton "procured me a Triangular glass-Prisme, to try therewith the celebrated Phaenomena of Colours" and opened up a new era in the scientific investigation of light [1]. It was not until 1801 that Johann Ritter discovered the ultraviolet (UV) region of the solar spectrum by showing that chemical action was caused by some form of energy in the dark portion beyond the violet [2]. In the previous year 1800, Sir William Herschel had demonstrated the existence of radiation beyond the red end of the visible spectrum, a component now known as infrared radiation [3].

These three components of the solar spectrum-ultraviolet, visible, and infrared-are referred to collectively as optical radiation. But it is the UV rays, constituting about $5 \%$ of terrestrial sunlight, that hold the greatest interest in photoimmunology. It is common

\footnotetext{
*Fax: +44-191-226-0970.

E-mail address: b.1.diffey@ncl.ac.uk.
}

practice to talk of ultraviolet light or UVL. This is incorrect; the term light should be reserved for those wavelengths of radiation (approximately $400-700 \mathrm{~nm}$ ) that reach the retina and result in a sensation of vision. The correct term is ultraviolet radiation or $U V R$.

\section{Nature of ultraviolet radiation}

UV radiation covers a small part of the electromagnetic spectrum. Other regions of this spectrum include radiowaves, microwaves, infrared radiation (heat), visible light, $\mathbf{X}$ rays, and $\gamma$ radiation. The feature that characterizes the properties of any particular region of the spectrum is the wavelength of the radiation. UV radiation spans the wavelength region from 400 to 100 $\mathrm{nm}$. Even in the UV portion of the spectrum the biological effects of the radiation vary enormously with wavelength and for this reason the UV spectrum is further subdivided into three regions. The notion to divide the UV spectrum into different spectral regions was first put forward at the Copenhagen meeting of the 
Second International Congress on Light held during August 1932. It was recommended that three spectral regions be defined as follows:

$$
\begin{array}{ll}
\text { UVA } & 400-315 \mathrm{~nm} \\
\text { UVB } & 315-280 \mathrm{~nm} \\
\text { UVC } & 280-100 \mathrm{~nm}
\end{array}
$$

The subdivisions are arbitrary and differ somewhat depending on the discipline involved. Environmental and dermatological photobiologists normally define the wavelength regions as

$$
\begin{array}{cc}
\text { UVA } & 400-320 \mathrm{~nm} \\
\text { UVB } & 320-290 \mathrm{~nm} \\
\text { UVC } & 290-200 \mathrm{~nm}
\end{array}
$$

The division between UVB and UVC is chosen as $290 \mathrm{~nm}$ since UV radiation at shorter wavelengths is unlikely to be present in terrestrial sunlight, except at high altitudes. The choice of $320 \mathrm{~nm}$ as the division between UVB and UVA is perhaps more arbitrary. Although radiation at wavelengths shorter than $320 \mathrm{~nm}$ is generally more photobiologically active than longerwavelength UV radiation, advances in molecular photobiology indicate that a subdivision at $330-340 \mathrm{~nm}$ may be more appropriate and for this reason the UVA region has, more recently, been divided into UVAI (340$400 \mathrm{~nm})$ and UVAII (320-340 $\mathrm{nm})$.

\section{Quantities and units}

Quantities of UV radiation are expressed using radiometric terminology (Table 1). Terms relating to a beam of radiation passing through space are radiant energy and radiant flux. Terms relating to a source of radiation are radiation intensity and radiance. The term irradiance, which is the most commonly used term in photobiology, relates to the object (e.g., patient) struck by the radiation. The radiometric quantities in Table 1 may also be expressed in terms of wavelength by adding the prefix spectral.

The time integral of the irradiance is strictly termed the radiant exposure, but is sometimes expressed as exposure dose or, even more loosely, as dose. The term dose

Table 1

Radiometric terms and units

\begin{tabular}{lll}
\hline Term & Unit & Symbol \\
\hline Wavelength & $\mathrm{nm}$ & $\lambda$ \\
Radiant energy & $\mathrm{J}$ & $Q$ \\
Radiant flux & $\mathrm{W}$ & $\phi$ \\
Radiant intensity & $\mathrm{W} \mathrm{sr}{ }^{-1}$ & $I$ \\
Radiance & $\mathrm{W} \mathrm{m}{ }^{-2} \mathrm{sr}^{-1}$ & $L$ \\
Irradiance & $\mathrm{W} \mathrm{m}^{-2}$ & $E$ \\
Radiant exposure & $\mathrm{J} \mathrm{m}{ }^{-2}$ & $H$ \\
\hline
\end{tabular}

in photobiology is analogous to the term energy fluence in radiobiology and not to absorbed dose. As yet the problems of estimating the energy absorbed by critical targets in the skin remain unsolved.

Although radiometric terminology is widely used in photobiology, the units chosen vary throughout the literature. For example, exposure doses may be quoted in $\mathrm{mJ} \mathrm{cm}{ }^{-2}$ or $\mathrm{kJ} \mathrm{m}^{-2}$. Table 2 summarizes the equivalence of these units.

\subsection{Radiometric calculations}

The most frequent radiometric calculation is to determine the time for which a patient (or other object), who is prescribed a certain dose (in $\mathrm{J} \mathrm{cm}^{-2}$ ), should be exposed when the radiometer indicates irradiance in $\mathrm{mW} \mathrm{cm}{ }^{-2}$. The relationship between these three quantities (time, dose, and irradiance) is simply

exposure time $(\mathrm{min})$

$$
=\frac{1000 \times \text { prescribed dose }\left(\mathrm{J} \mathrm{cm}^{-2}\right)}{60 \times \text { measured irradiance }\left(\mathrm{mW} \mathrm{cm}^{-2}\right)} .
$$

\subsection{The standard erythema dose}

The problem of dosimetry in photodermatology lies in the fact that the ability of UV radiation to elicit erythema in human skin depends strongly on wavelength, encompassing a range of four orders of magnitude between 250 and $400 \mathrm{~nm}$. Thus a statement that a subject received an exposure dose of $1 \mathrm{~J} \mathrm{~cm}^{-2}$ of UV radiation conveys nothing about the consequences of that exposure in terms of erythema. If the radiation source were a UVA fluorescent lamp, no erythemal response would be seen apart from that in people exhibiting severe, abnormal pathological photosensitivity. The same dose delivered from an unfiltered mercury arc lamp or fluorescent sunlamp would result in marked violaceous erythema in most white-skinned individuals. Consequently, there is often a need to express the exposure as an erythemally weighted quantity.

It has been common practice for many years to use the term minimal erythema dose (MED) as a "measure" of erythemal radiation. This is absurd because the MED is not a standard measure of anything but, on the contrary, encompasses the variable nature of individual sensitivity to UV radiation.

Table 2

Equivalent radiometric quantities

\begin{tabular}{lll}
\hline To convert from & To & Multiply by \\
\hline $\mathrm{J} \mathrm{cm}^{-2}$ & $\mathrm{~mJ} \mathrm{~cm}{ }^{-2}$ & $10^{3}$ \\
$\mathrm{~J} \mathrm{~cm}^{-2}$ & $\mathrm{~J} \mathrm{~m}^{-2}$ & $10^{4}$ \\
$\mathrm{~J} \mathrm{~m}^{-2}$ & $\mathrm{~mJ} \mathrm{~cm}^{-2}$ & $10^{7}$ \\
$\mathrm{~kJ} \mathrm{~m}^{-2}$ & $\mathrm{~J} \mathrm{~cm}^{-2}$ & $10^{7}$ \\
$\mathrm{~kJ} \mathrm{~m}^{-2}$ & $\mathrm{~mJ} \mathrm{~cm}$ & $10^{10}$ \\
\hline
\end{tabular}


To avoid further confusing abuse of the term $M E D$, it has been proposed [4] that this term be reserved solely for observational studies in humans and other animals. The term Standard Erythema Dose (SED) should be used to refer to erythemal effective radiant exposures from natural and artificial sources of UV radiation. One SED is equivalent to an erythemal effective radiant exposure of $100 \mathrm{~J} \mathrm{~m}^{-2}$ [5]. Examples of how the SED can be used are:

- The ambient diurnal exposure on a clear sky summer day in Europe is approximately 30-40 SED.

- An exposure dose of 4 SED would be expected to produce moderate erythema on unacclimated white skin, but minimal or no erythema on previously exposed skin.

\section{Production of ultraviolet radiation}

UV radiation is produced either by heating a body to an incandescent temperature, as is the case with solar UV, or by passing an electric current through a gas, usually vaporized mercury. The mercury atoms become excited by collisions with the electrons flowing between the lamp's electrodes. The excited electrons return to particular electronic states in the mercury atom and in doing so release some of the energy they have absorbed in the form of optical radiation, that is, ultraviolet, visible, and infrared radiation.

\subsection{Spectral power distribution}

We talk loosely of "UVA lamps" or "UVB lamps." However, such a label does not characterize UV lamps adequately since nearly all lamps used in photobiology will emit UVA and UVB, and even UVC, visible light, and infrared radiation. The only correct way to specify the nature of the emitted radiation is by reference to the spectral power distribution. This is a graph (or table) that indicates the radiated power as a function of wavelength. Fig. 1 shows the spectral power distribution of UV radiation emitted by three common fluorescent lamps used for the photo(chemo)therapy of skin disease.

\section{Solar ultraviolet radiation}

The spectrum of extraterrestrial solar radiation approximates to a blackbody of about $5800 \mathrm{~K}$. The irradiance of solar radiation outside the atmosphere but at the earth's mean distance from the sun is termed the solar constant and is $1.37 \mathrm{~kW} \mathrm{~m}^{-2}$. Of this, about $9 \%$ is in the ultraviolet $(\lambda<400 \mathrm{~nm})$. The solar output is not constant but varies with a 27 -day apparent solar rotation and an 11-year cycle of sunspot activity. The variability affects mostly those wavelengths that are absorbed in the atmosphere $(\lambda<290 \mathrm{~nm})$ and the effect on terrestrial UVB and UVA is minimal. Because of the elliptical orbit of the sun, the sun-earth distance varies by about $3.4 \%$ from a minimum on the perihelion (about January 3 ) to a maximum on the aphelion (about July 5). This results in a variation in intensity of about $7 \%$ and results in slightly higher UV levels in Southern Hemisphere summers than in the Northern Hemisphere.

\subsection{Solar elevation}

Both the quality (spectrum) and quantity (intensity) of terrestrial UV radiation vary with the elevation of the sun above the horizon, or solar altitude. (The complementary angle between the sun and the local vertical is termed the solar zenith angle.) The solar altitude depends on the time of day, day of year, and geographical location (latitude and longitude). The intensity changes because as the solar zenith angle increases the number of UV rays emitted by the sun into a given solid angle is distributed over a larger area on the earth's surface. If we neglect absorption in the atmosphere, the intensity at a solar zenith of $\theta^{\circ}$ is simply equal to the intensity with the sun directly overhead (solar zenith of $0^{\circ}$ ) multiplied by cosine $\theta$.

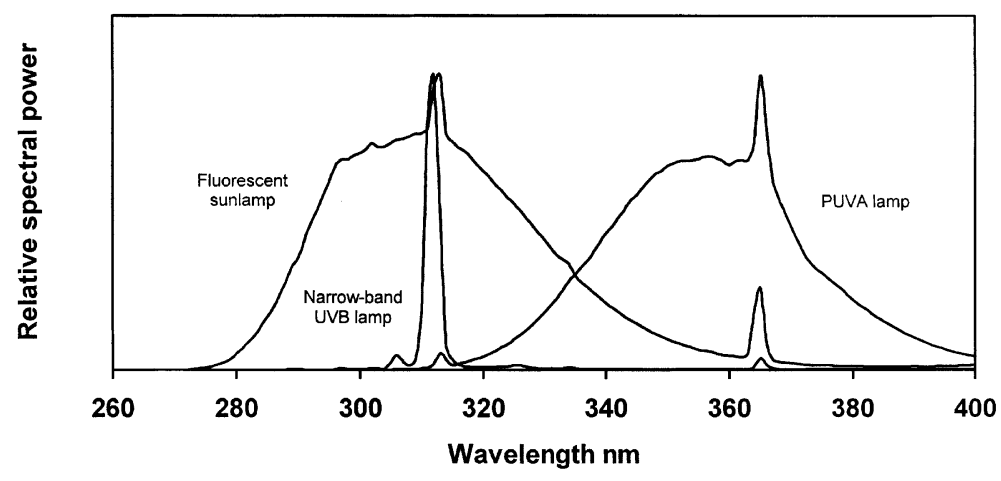

Fig. 1. Spectral power distribution of three common lamps used in phototherapy. 
We cannot, however, neglect attenuation of the sun's UV rays in the atmosphere, particularly by ozone (see below), and this serves to absorb UV in a wavelengthdependent manner and hence change the spectrum relative to extraterrestrial sunlight. As the sun falls lower in the sky, the path length of the sun's UV rays through the atmosphere increases and as a consequence the intensity of UV reaching the earth's surface decreases at all wavelengths, particularly those shorter than $320 \mathrm{~nm}$. On a summer's day, UVB (when taken as $290-320 \mathrm{~nm}$ ) constitutes approximately $6 \%$ of terrestrial UV, and UVA (when taken as $320-400 \mathrm{~nm}$ ), the remaining $94 \%$. But since UVB is much more effective than UVA at causing biological damage, solar UVB contributes about $80 \%$ toward most of the harmful effects we associate with sun exposure, with solar UVA contributing the remaining $20 \%$.

\subsection{Atmospheric attenuation}

The quality and quantity of solar UV are modified as the sun's rays pass through the atmosphere. The principal interactions in the stratosphere $(\sim 10-50 \mathrm{~km}$ above sea level) are absorption by ozone and scattering by molecules such as $\mathrm{N}_{2}$ and $\mathrm{O}_{2}$. In the troposphere ( 0 to $\sim 10 \mathrm{~km}$ above sea level) absorption by pollutants such as ozone, $\mathrm{NO}_{2}$, and $\mathrm{SO}_{2}$ and scattering by particulates (e.g., soot) and clouds are the main attenuating processes.

\subsection{Clouds}

Since pure water is a very weak absorber of UV radiation, clouds, which are composed of either liquid or ice droplets, attenuate UV primarily by scattering. Cloud droplets have radii from about 1 to $30 \mu \mathrm{m}$, considerably larger than UV wavelengths, and as a consequence, clouds attenuate UVB and UVA to much the same extent. Clouds reduce UV intensity, although not to the same extent as infrared intensity. This is because water in clouds attenuates solar infrared much more than UV and so the risk of overexposure is increased because the warning sensation of heat is diminished. Roughly speaking the ambient annual UV radiation is about two-thirds that estimated for clear skies in temperate latitudes, rising to about $75 \%$ for the tropics.

\subsection{Surface reflection}

Reflection of solar UV radiation from most ground surfaces is normally less than $10 \%$. The main exceptions are gypsum sand, which reflects about $15-30 \%$, and snow, which can reflect up to $90 \%$. Contrary to popular belief, calm water reflects only about $5 \%$ of incident UV radiation, although up to $20 \%$ is reflected from choppy water. Because UV rays pass easily through water, swimming in either the sea or open-air pools offers little protection against sunburn.

\subsection{Reference sunlight spectrum}

It is clear from the above that the spectrum of sunlight is changing continuously. However, for many purposes, such as calculating the protection provided by a topical sunscreen, it is necessary to use some reference spectrum. Two spectra that are often employed in such calculations are those measured on a clear summer's day around noon at Melbourne $\left(38^{\circ} \mathrm{S}\right)$ and Albuquerque $\left(38^{\circ} \mathrm{N}\right)$. Numerical values of the spectral irradiance from 290 to $400 \mathrm{~nm}$ in 1-nm steps are given in Table 3.

\subsection{Ultraviolet climatology}

The sun's UV rays are strongest in the 4-h period around local noon when $50-60 \%$ of a summer's day UV is received. Table 4 summarizes the percentage of ambient UV radiation present at different times during a summer's day. The data are applicable to all latitudes between tropical and temperate, i.e., $20-60^{\circ}$, and assume that solar noon occurs at 1:00 PM. So someone going in the sun between 10:30 and 11:30 $\mathrm{AM}$ and again from $4: 30 \mathrm{PM}$ until the end of the day avoids $100-(12+4+2+1)=81 \%$ of the ambient available.

The variation of UVB and UVA during a clear summer's day, and throughout the year, in the United Kingdom is shown in Figs. 2 and 3, respectively.

\section{Simulated sources of sunlight}

\subsection{Xenon arc lamps}

For many experimental studies in photobiology it is simply not practicable to use natural sunlight and so artificial sources of UV radiation designed to simulate the UV component of sunlight are employed. No such source will match exactly the spectral power distribution of sunlight and as the shorter UV wavelengths (less than around $340 \mathrm{~nm}$ ) are generally more photobiologically active than longer UV wavelengths, the usual goal is to match as closely as possible the UVB and UVAII regions.

The classic so-called solar simulator consists of an optically filtered xenon arc lamp. This lamp has a smooth continuous spectrum in the UV region and various models of solar simulators are available with input power in the range $75 \mathrm{~W}$ to $6 \mathrm{~kW}$ and above, from companies that include Oriel Corporation, Solar Light, Spectral Energy Corporation, and Schoeffel Optical [8]. Optical filters and dichroic mirrors are used to shape the spectrum. In most cases a 1-mm-thick Schott Type WG320 filter is used to control the short wavelength end 
Table 3

Summer solar spectral irradiance at noon on clear days

\begin{tabular}{|c|c|c|c|c|c|c|c|c|}
\hline $\begin{array}{l}\lambda \\
(\mathrm{nm})\end{array}$ & $\begin{array}{l}38^{\circ} \mathrm{N}^{\mathrm{a}} \\
\left(\mathrm{Wm}^{-2} \mathrm{~nm}^{-1}\right)\end{array}$ & $\begin{array}{l}38^{\circ} \mathrm{S}^{\mathrm{b}} \\
\left(\mathrm{Wm}^{-2} \mathrm{~nm}^{-1}\right)\end{array}$ & $\begin{array}{l}\lambda \\
(\mathrm{nm})\end{array}$ & $\begin{array}{l}38^{\circ} \mathrm{N}^{\mathrm{a}} \\
\left(\mathrm{Wm}^{-2} \mathrm{~nm}^{-1}\right)\end{array}$ & $\begin{array}{l}38^{\circ} \mathrm{S}^{\mathrm{b}} \\
\left(\mathrm{Wm}^{-2} \mathrm{~nm}^{-1}\right)\end{array}$ & $\begin{array}{l}\lambda \\
(\mathrm{nm})\end{array}$ & $\begin{array}{l}38^{\circ} \mathrm{N}^{\mathrm{a}} \\
\left(\mathrm{Wm}^{-2} \mathrm{~nm}^{-1}\right)\end{array}$ & $\begin{array}{l}38^{\circ} \mathrm{S}^{\mathrm{b}} \\
\left(\mathrm{Wm}^{-2} \mathrm{~nm}^{-1}\right)\end{array}$ \\
\hline 290 & 0.00000 & 0.00006 & 327 & 0.473 & 0.618 & 364 & 0.648 & 0.808 \\
\hline 291 & 0.00002 & 0.00009 & 328 & 0.501 & 0.566 & 365 & 0.683 & 0.766 \\
\hline 292 & 0.00003 & 0.00019 & 329 & 0.517 & 0.629 & 366 & 0.718 & 0.967 \\
\hline 293 & 0.00016 & 0.00027 & 330 & 0.532 & 0.708 & 367 & 0.740 & 0.911 \\
\hline 294 & 0.00029 & 0.00047 & 331 & 0.533 & 0.612 & 368 & 0.762 & 0.861 \\
\hline 295 & 0.00079 & 0.00093 & 332 & 0.533 & 0.632 & 369 & 0.764 & 0.872 \\
\hline 298 & 0.00337 & 0.00485 & 335 & 0.514 & 0.667 & 372 & 0.750 & 0.814 \\
\hline 299 & 0.00601 & 0.00890 & 336 & 0.504 & 0.575 & 373 & 0.706 & 0.787 \\
\hline 300 & 0.00864 & 0.01110 & 337 & 0.502 & 0.536 & 374 & 0.661 & 0.705 \\
\hline 301 & 0.01612 & 0.0196 & 338 & 0.499 & 0.617 & 375 & 0.664 & 0.685 \\
\hline 302 & 0.02360 & 0.0235 & 339 & 0.519 & 0.660 & 376 & 0.666 & 0.845 \\
\hline 303 & 0.03355 & 0.0513 & 340 & 0.539 & 0.765 & 377 & 0.706 & 0.876 \\
\hline 308 & 0.0968 & 0.135 & 345 & 0.535 & 0.640 & 382 & 0.642 & 0.693 \\
\hline 309 & 0.115 & 0.127 & 346 & 0.534 & 0.642 & 383 & 0.614 & 0.543 \\
\hline 310 & 0.134 & 0.147 & 347 & 0.536 & 0.680 & 384 & 0.585 & 0.587 \\
\hline 311 & 0.155 & 0.235 & 348 & 0.537 & 0.638 & 385 & 0.605 & 0.834 \\
\hline 312 & 0.175 & 0.215 & 349 & 0.548 & 0.640 & 386 & 0.626 & 0.724 \\
\hline 313 & 0.194 & 0.246 & 350 & 0.559 & 0.724 & 387 & 0.649 & 0.775 \\
\hline 314 & 0.213 & 0.269 & 351 & 0.574 & 0.743 & 388 & 0.672 & 0.765 \\
\hline 315 & 0.228 & 0.283 & 352 & 0.589 & 0.717 & 389 & 0.715 & 0.795 \\
\hline 316 & 0.243 & 0.243 & 353 & 0.601 & 0.695 & 390 & 0.757 & 0.948 \\
\hline 317 & 0.261 & 0.371 & 354 & 0.613 & 0.829 & 391 & 0.737 & 1.03 \\
\hline 318 & 0.279 & 0.316 & 355 & 0.608 & 0.832 & 392 & 0.716 & 0.948 \\
\hline 319 & 0.297 & 0.353 & 356 & 0.603 & 0.757 & 393 & 0.686 & 0.494 \\
\hline
\end{tabular}

${ }^{a}$ Measured in Albuquerque $\left(38^{\circ} \mathrm{N}\right)$ at noon on 3 July. Measurements were made at 2-nm intervals and interpolated values have been added here to give points every $1 \mathrm{~nm}[6]$.

${ }^{\mathrm{b}}$ Measured in Melbourne (38 $\mathrm{S}$ ) at solar noon on 17 January 1990. Measurements were made at the Australian Radiation Laboratory with a Spex 1680B double monochromator with a resolution of $1 \mathrm{~nm}$ [7].

Table 4

Approximate percentage of ambient UV received during a clear summer's day from tropical $\left(20^{\circ}\right)$ to temperate $\left(60^{\circ}\right)$ latitudes ${ }^{\mathrm{a}}$

\begin{tabular}{lc}
\hline Hourly interval & \% Daily UV \\
\hline Before 9:30 AM & 6 \\
9:30 A M to $10: 30 \mathrm{AM}$ & 8 \\
10:30 AM to $11: 30 \mathrm{AM}$ & 12 \\
11:30 A M to $12: 30 \mathrm{PM}$ & 15 \\
12:30 PM to $1: 30 \mathrm{PM}$ & 17 \\
1:30 PM to $2: 30 \mathrm{PM}$ & 15 \\
2:30 PM to $3: 30 \mathrm{PM}$ & 12 \\
3:30 PM to $4: 30 \mathrm{PM}$ & 8 \\
4:30 PM to $5: 30 \mathrm{PM}$ & 4 \\
5:30 PM to $6: 30 \mathrm{PM}$ & 2 \\
After $6: 30 \mathrm{PM}$ & 1 \\
\hline
\end{tabular}

\footnotetext{
${ }^{\mathrm{a}}$ Solar noon is assumed to occur at 1:00 PM.
}

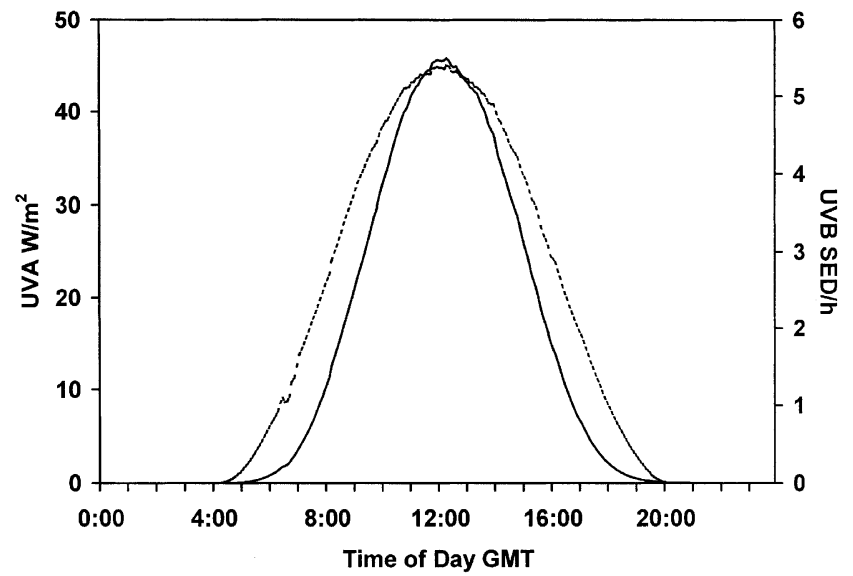

Fig. 2. Variation of ambient UVB (solid line) and UVA (broken line) during a clear summer day in the United Kingdom. 


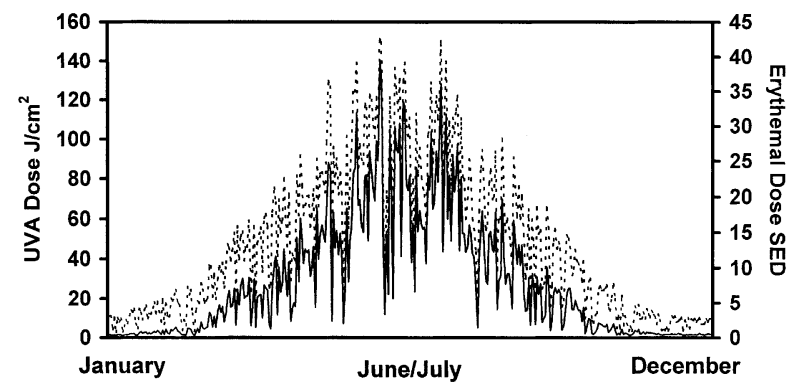

Fig. 3. Diurnal variation of ambient UVB (solid line) and UVA (broken line) throughout 1994 measured at Durham, United Kingdom (latitude $55^{\circ} \mathrm{N}$ ).

of the spectrum. By varying the thickness of the filter from 1 to 1.5 or $2 \mathrm{~mm}$, spectra are obtained that approximate varying solar altitudes. The simulator normally also incorporates an UV-transmitting, visible light absorbing filter (e.g., Schott UG5 or UG11) or other filters or multiple dichroic mirrors to remove visible and infrared wavelengths. The spectrum of a solar simulator is compared with natural sunlight in Fig. 4. A comprehensive review of solar simulators, with specific reference to sunscreen testing, is given by Wilkinson [8].

\subsection{Fluorescent lamps}

A drawback of arc lamp solar simulators is that the irradiation field is generally limited to less than around $15 \times 15 \mathrm{~cm}$, although it is possible to achieve uniform flux over a larger area albeit with a reduction in irradiance. This may pose little problem if the object is to irradiate small areas of skin but for studies where large numbers of experimental animals or plants are to be irradiated, the limited irradiation field is a real problem. Because of this attention has turned to fluorescent lamps as sources of simulated UV [9]. One way to evaluate candidate lamps and decide which is the most appropriate approximation to sunlight is to calculate the percentage relative cumulative erythemal effectiveness

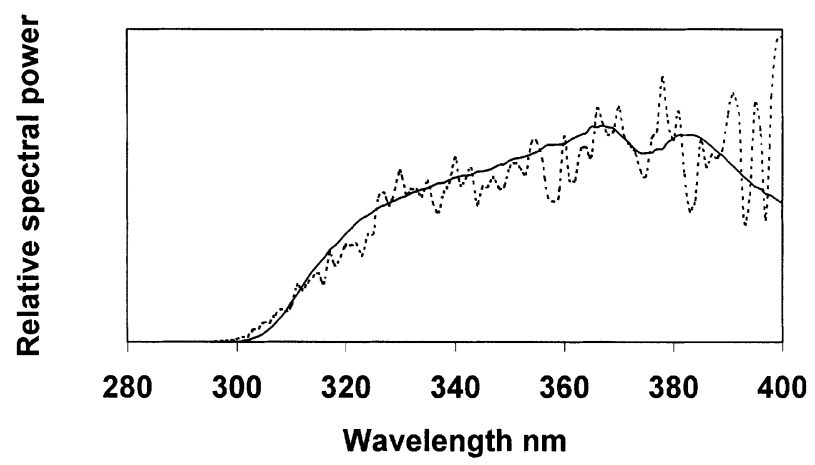

Fig. 4. Spectral power distribution of clear sky, terrestrial UV radiation measured at around noon in summer at a latitude of $38^{\circ} \mathrm{S}$ (broken line) and a xenon arc filtered with a WG320 (2 mm thick) and UG5 (1 mm thick) filter (solid line).
(\%RCEE) for a number of wavebands and to compare these values with the \%RCEE values of a "standard sun" [10]. The \%RCEE for the spectral range 290 to $\lambda_{\mathrm{c}}$ is the erythemally effective UV radiation within this waveband expressed as a percentage of the total erythemally effective radiation from 290 to $400 \mathrm{~nm}$. This is calculated mathematically as

$\% \mathrm{RCEE}=100 \times \sum_{290}^{\lambda_{\mathrm{c}}} E(\lambda) \varepsilon(\lambda) \Delta \lambda / \sum_{290}^{400} E(\lambda) \varepsilon(\lambda) \Delta \lambda$.

$E(\lambda)$ is the relative spectral power distribution of the UV source and $\varepsilon(\lambda)$ is the effectiveness of radiation of wavelength $\lambda \mathrm{nm}$ in producing erythema in human skin [5]. Table 5 compares \%RCEE values from a number of fluorescent lamps with lower and upper acceptance limits for a "standard sun" given by the European Cosmetic, Toiletry and Perfumery Association (COLIPA) [10]. It can be seen that the Arimed B lamp is perhaps the best choice as a source of simulated solar UV radiation from those given, although there is little to choose between this lamp and some of the others. The TL-12 (equivalent spectrum to the Westinghouse sunlamp), a mainstay of photobiological research for many years, is a poor surrogate for solar UV radiation.

\section{Monochromatic radiation}

A fundamental investigation in photoimmunology is the study of how efficient different wavelengths of UV radiation are in causing an observable effect, such as depletion of Langerhans cells. This demands irradiating subjects with monochromatic radiation of varying wavelength. The term monochromatic is, in fact, misleading since the spectral distribution of radiation emerging from the exit slit of an irradiation monochromator or transmitted by an interference filter is approximately triangular. The width of the distribution is a function of the entrance and exit slit widths of the monochromator or the design of the interference filter.

\subsection{Interference filters}

An interference filter transmits a narrow band of wavelengths, while prohibiting the transmission of wavelengths outside the desired waveband. These filters are available at a wide range of peak wavelengths and bandwidths.

\subsection{Irradiation monochromator}

This is the ideal tool for action spectroscopy in photoimmunology since it comprises virtually an infinite selection of central wavelength and bandwidth. The source of UV radiation is normally a xenon arc lamp, 
Table 5

UVB and UVA components and percentage relative cumulative erythemal effectiveness (\%RCEE) for the summer sun and a number of fluorescent lamps

\begin{tabular}{|c|c|c|c|c|c|c|}
\hline & Sun $^{\mathrm{a}}$ & $\mathrm{A}^{\mathrm{b}}$ & $\mathrm{B}$ & $\mathrm{C}$ & $\mathrm{D}$ & $\mathrm{E}$ \\
\hline$\%$ UVB (290-315 nm) & $3.35 \%$ & $55.64 \%$ & $2.58 \%$ & $4.54 \%$ & $4.30 \%$ & $3.43 \%$ \\
\hline \%UVA (315-400 nm) & $96.65 \%$ & $44.36 \%$ & $97.42 \%$ & $95.46 \%$ & $95.70 \%$ & $96.57 \%$ \\
\hline$<290 \mathrm{~nm}(<1.0 \%)$ & $0.047 \%$ & $19.6 \%$ & $0.087 \%$ & $0.095 \%$ & $0.000 \%$ & $0.089 \%$ \\
\hline $290-310 \mathrm{~nm}(46.0-67.0 \%)$ & $62.3 \%$ & $77.6 \%$ & $51.4 \%$ & $60.7 \%$ & $42.8 \%$ & $53.4 \%$ \\
\hline $290-320 \mathrm{~nm}(80.0-91.0 \%)$ & $86.4 \%$ & $80.2 \%$ & $79.2 \%$ & $86.7 \%$ & $80.9 \%$ & $81.9 \%$ \\
\hline $290-350 \mathrm{~nm}(93.5-99.0 \%)$ & $95.8 \%$ & $80.4 \%$ & $94.5 \%$ & $97.1 \%$ & $96.4 \%$ & $95.9 \%$ \\
\hline
\end{tabular}

\footnotetext{
${ }^{\mathrm{a}}$ Melbourne summer sun (see Table 3).

b Lamp A: TL-12 (“fluorescent sunlamp"), Philips Lighting, The Netherlands; lamp B: Bellarium S, Wolff System, Germany; lamp C: Arimed B, Cosmedico, Germany; lamp D: CLEO Natural, Philips Lighting; lamp E: UVA-340; Q-Panel Lab Products, Cleveland, OH.
}

principally because of its continuous spectral emission. Apart from cost, the main drawback of an irradiation monochromator is the attention that must be paid to measuring and understanding factors such as output irradiance, wavelength accuracy and stray radiation if scientifically meaningful results are to be obtained. Further information on the technical performance of irradiation monochromators is readily available [11].

\section{Dosimetry of ultraviolet radiation}

Dosimetry is the science of radiation measurement. There are two principal reasons why UV radiation should be measured: to allow consistent radiation exposure of patients, animals, cells, or plants over many months and years within a local laboratory; and to allow the results of irradiations made in different laboratories to be published and compared.

It is important to distinguish between these two objectives. The first requires precision, or reproducibility. The radiometer is used as a monitor to give a reference measurement and so it needs to be stable. Accuracy, that is, absolute calibration against some accepted standard, is not essential. The second objective requires both precision and accuracy. Here the radiometer must not only be stable from one day to the next, but also the display (in, say, $\mathrm{mW} \mathrm{cm} \mathrm{cm}^{-2}$ ) must be traceable to absolute standards. Although electro-optical technology has improved over the years, resulting in the availability of versatile and precise UV radiometric equipment, these improvements have not been accompanied by improved accuracy due to misunderstandings about calibration.

\section{Spectroradiometry}

Spectroradiometry is the technique for measuring the spectral power distribution (relative measurement showing shape of spectrum) or spectral irradiance (absolute measurement showing shape and power) of a source of optical radiation. The three basic requirements of a spectrometer system are the input optics, designed to conduct the radiation from the source into the monochromator, which disperses the radiation onto a detector.

\subsection{Input optics}

The spectral transmission characteristics of monochromators depend on the angular distribution and polarization of the incident radiation as well as the position of the beam on the entrance slit. For measurement of spectral irradiance, particularly from extended sources such as linear arrays of fluorescent lamps or daylight, direct irradiation of the entrance slit should be avoided. There are two types of input optics available to ensure that the radiation from different source configurations is depolarized and follows the same optical path through the system-the integrating sphere or the diffuser.

\subsection{Monochromator}

A blazed ruled diffraction grating is normally preferred to a prism as the dispersion element in the monochromator used in a spectroradiometer, mainly because of better stray radiation characteristics. Highperformance spectroradiometers, used for determining low UV spectral irradiances in the presence of high irradiances at longer wavelengths, demand extremely low stray radiation levels. Such systems may incorporate a double monochromator, that is, two single-ruled-grating monochromators in tandem.

\subsection{Detector}

Photomultiplier tubes, incorporating a photocathode with an appropriate spectral response, are normally the 
detectors of choice in spectroradiometers. However, if radiation intensity is not a problem, solid-state photodiodes may be used, since they require simpler and cheaper electronic circuitry.

\section{Calibration of spectroradiometers}

It is important that spectroradiometers are calibrated over the wavelength range of interest using standard lamps [12]. A tungsten filament lamp operating at a color temperature $^{1}$ of about $3000 \mathrm{~K}$ can be used as a standard lamp for the spectral interval $250-2500 \mathrm{~nm}$, although workers concerned solely with the UV region $(200-400 \mathrm{~nm})$ may prefer to use a deuterium lamp.

\subsection{Sources of error in spectroradiometry}

Accurate spectroradiometry, even where only relative spectral power distributions are used, requires careful attention to detail [12]. Factors that can affect accuracy include wavelength calibration, bandwidth, stray radiation, polarization, angular dependence, linearity, and calibration sources.

\section{Commercial spectroradiometers}

Modern spectroradiometers (e.g., Model OL754, Optonic Laboratories, Orlando, FL) incorporate a number of features that include:

- Automated computer control of data collection and display.

- Wavelength accuracy of typically $\pm 0.2 \mathrm{~nm}$ over the spectral range $200-1600 \mathrm{~nm}$.

- Low stray light rejection level of $1 \times 10^{-8}$ at $285 \mathrm{~nm}$ by using a double holographic grating monochromator in combination with an order blocking filter wheel.

- High sensitivity and wide dynamic range.

- User selectable bandwidths.

The above type of spectroradiometer operates by stepwise scanning through the required wavelength range at scan speeds of $0.1-2 \mathrm{~nm}$ per second. By using a diode array as the detector in conjunction with a single grating spectrograph, instantaneous spectral power distributions can be obtained in much more compact and portable systems (e.g., Solatell, 4D Controls, Redruth, Cornwall, UK). What such a device gains in speed, cost, and portability, it loses in performance in terms of stray light rejection, which is typically at a level of no better than $1 \times 10^{-4}$ at $285 \mathrm{~nm}$. This is particularly important

\footnotetext{
${ }^{1}$ The color temperature is the temperature at which a blackbody has the same spectral power distribution in the visible region (i.e., gives the same impression of color) as the lamp in question.
}

in the spectroradiometry of solar UVB (wavelengths less than $315 \mathrm{~nm}$ ), but may not be problem in the spectral characterization of UVA lamps in studies where the investigator believes the small UVB (and possibly UVC) component is of no biological significance, possibly because of optical filtering.

\section{Narrowband radiometry}

Although spectroradiometry is the fundamental way to characterize the radiant emission from a light source, radiation output is normally measured by techniques of narrowband radiometry. Narrowband radiometers generally combine a detector (such as a vacuum phototube or a solid-state photodiode) with a wavelength-selective device (such as a color glass filter or interference filter) and suitable input optics (such as a quartz hemispherical diffuser or polytetrafluoroethylene (PTFE) window).

\subsection{Spectral sensitivity}

To meet the criterion for a UVB radiometer, say, the sensor should have a uniform spectral response from 280 to $315 \mathrm{~nm}$ (the UVB waveband) with zero response outside this interval. In other words, the electrical output from the sensor should depend only on the total power within the UVB waveband received by the sensor and not on how the power is distributed with respect to wavelength. In practice no such sensor exists with this ideal spectral response (neither does one exist that measures UVA or UVC correctly for that matter). All radiometers that combine a photodetector with an optical filter have a nonuniform spectral sensitivity within their normal spectral band. Consequently it is important that narrowband radiometers are calibrated spectroradiometrically for every type of UV source (where type refers to the spectral power distribution) that it is proposed to measure [13].

\subsection{Angular response}

Narrowband radiometers are often used to measure the irradiance from extended sources of radiation such as linear fluorescent lamps or the sky. In these instances it is important that the sensor "sees" radiation coming from all parts of the source, and does not have a limited field of view; that is, the sensor is not collimated. Furthermore, the sensor should have a cosine-weighted response; this means that a sensor that is measuring irradiance on a plane must weight the incident flux by the cosine of the angle between the incoming radiation and the normal to the surface.

In practice it is very difficult to achieve a perfect cosine-weighted response, but sensors incorporating a 
PTFE or quartz input optic can get very close and only diverge significantly from a cosine-weighted response at angles exceeding $70^{\circ}$ from the normal.

\section{Broadband radiometry}

Broadband radiometry uses a detector that responds equally to all wavelengths of optical radiation. The most common detector used is the thermopile and this is especially useful in measuring the irradiance from an irradiation monochromator used in the investigation of skin photosensitivity. Until a few years ago, commercial thermopiles were handmade, expensive, and fragile devices. A major advance came with the production of multiple junction thermopiles based on thin-film technology. These devices are rugged and much less expensive and typified by the Dexter range of thermopiles (Dexter Research Center, MI), which have found a role in dermatological photobiology [14].

Thermopiles measure absolute radiant power and calibration can only be achieved satisfactorily by national standards laboratories, such as the National Physical Laboratory in the United Kingdom [13].

\section{Radiometer stability}

It should be remembered that the sensitivity of all radiometers changes with time; frequent exposure to high-intensity sources of optical radiation accelerate this change. For this reason it is always sound policy to acquire two radiometers, preferably of the same type, one of which has a calibration traceable to a national standards laboratory. This radiometer should be reserved solely for intercomparisons with the other radiometer(s) used for routine purposes. A measurement of the same source is made with each radiometer and a ratio calculated. It is the stability of this ratio over a period of months and years that indicates long-term stability and good precision.

\section{Measuring personal exposure to ultraviolet radiation}

Personal exposure to UV radiation can be measured using physical, chemical, or biological dosimeters.

\subsection{Physical dosimeters}

The availability of miniature electro-optical UV sensors means that it is possible to construct small UV detectors that can be electrically coupled to a portable data logger carried in a trouser pocket, worn on a belt, or clipped to spectacles. By this means it is possible to record UV exposure on a second-by-second basis, which permits a clearer understanding of human behavior in sunlight [15-17].

\subsection{Chemical dosimeters}

The use of chemical methods, which measure the chemical change produced by the radiation, is called actinometry. These techniques usually form the basis of personal UV dosimeters. The most commonly used material for studies of personal UV dosimetry has been the thermoplastic polysulfone. The basis of the method is that when film is exposed to UV radiation at wavelengths principally in the UVB waveband, its UV absorption increases. The increase in absorbance measured at a wavelength of $330 \mathrm{~nm}$ increases with UV dose [18]. In practice the film $(40-50 \mu \mathrm{m}$ thick $)$ is mounted in cardboard or plastic photographic holders. Applications of UV dosimetry with polysulfone film have included:

sun exposure of children,

sun exposure from different leisure pursuits, sun exposure from different occupations, anatomical distribution of sunlight in humans and animals,

clinical photosensitivity studies,

UV exposure of patients from therapeutic light sources,

UV exposure of workers in industry.

\subsection{Biological dosimeters}

Biological techniques of measurement are generally limited to the use of viruses and microorganisms. The bacteriophage $\mathrm{T} 7$ has been described for use as a UV biosensor [19]. It has been used to monitor ambient UV radiation, and when combined with an appropriate optical filter, a spectral response similar to the action spectrum for erythema in human skin can be achieved [20].

\section{References}

[1] I. Newton, Philos. Trans. R. Soc. London 6 (1672) 3075-3087.

[2] J.W. Ritter, Phys.-Chem. Abh. vol. 2, Leipzig, 1801.

[3] W. Herschel, Philos. Trans. R. Soc. London 90 (1800) 255-326.

[4] B.L. Diffey, C.T. Jansén, F. Urbach, H.C. Wulf, Photodermatol. Photoimmunol. Photomed. 13 (1997) 64-66.

[5] CIE Standard. Erythema reference action spectrum and standard erythema dose. CIE S 007/E-1998. Commission Internationale de l'Éclairage, Vienna, 1998.

[6] R.M. Sayre, C. Cole, W. Billhimer, J. Stanfield, R.D. Ley, Photodermatol. Photoimmunol. Photomed. 7 (1990) 159-165.

[7] H.P. Gies, C.R. Roy, A. McLennan, B.L. Diffey, M. Pailthorpe, C. Driscoll, M. Whillock, A.F. McKinlay, K. Grainger, I. Clark, R.M. Sayre, Health Phys. 73 (1997) 456-464.

[8] F. Wilkinson, in: R. Matthes, D. Sliney (Eds.), Measurements of Optical Radiation Hazards, International Commission on Nonionizing Radiation Protection, 1998, pp. 653-684. 
[9] D.B. Brown, A.E. Peritz, D.L. Mitchell, S. Chiarello, J. Uitto, F.P. Gasparro, Photochem. Photobiol. 72 (2000) 340-344.

[10] COLIPA. Sun Protection Factor Method, European Cosmetic Toiletry, and Perfumery Association COLIPA, Brussels, Belgium, 1994.

[11] B.L. Diffey, in: Ultraviolet Radiation in Medicine, IoP, Bristol, 1982, pp. 47-59.

[12] R. Saunders, in: R. Matthes, D. Sliney (Eds.), Measurements of Optical Radiation Hazards, International Commission on NonIonizing Radiation Protection, 1998, pp. 473-482.

[13] T. Goodman, in: R. Matthes, D. Sliney (Eds.), Measurements of Optical Radiation Hazards, International Commission on NonIonizing Radiation Protection, 1998, pp. 589-602.

[14] P.J. Mountford, V.J. Davies, Clin. Phys. Physiol. Meas. 8 (1987) $325-330$.
[15] B.L. Diffey, P.J. Saunders, Photochem. Photobiol. 61 (1995) 615618.

[16] D.D. Duncan, W. Schneider, K.J. West, S.J. Kirkpatrick, S.K. West, Photochem. Photobiol. 62 (1995) 94-100.

[17] P. Autier, J.-F. Doré, A.C. Reis, A. Grivegnée, L. Ollivaud, F. Truchetet, E. Chamoun, N. Rotmensz, G. Severi, J.-P. Césarini, Br. J. Cancer 83 (2000) 1243-1248.

[18] B.L. Diffey, in: B.L. Diffey (Ed.), Radiation Measurement in Photobiology, Academic Press, London, 1989, pp. 135159.

[19] G. Rontó, S. Gáspár, A. Bérces, J. Photochem. Photobiol. 12 (1992) 285-294.

[20] L.E. Quintern, Y. Furusawa, K. Fukutsu, H. Holtschmidt, J. Photochem. Photobiol. 37 (1997) 158-166. 